Mots. Les langages du politique

Trente ans d'étude des langages du politique

(1980-2010)

\title{
Analyser le discours politique en Allemagne (1980-2010)
}

Johannes Angermuller

\section{(2) OpenEdition}

\section{Journals}

Édition électronique

URL : https://journals.openedition.org/mots/19884

DOI : $10.4000 /$ mots. 19884

ISSN : 1960-6001

Éditeur

ENS Éditions

Édition imprimée

Date de publication : 30 novembre 2010

Pagination : 183-189

ISBN : 978-2-84788-235-3

ISSN : 0243-6450

Référence électronique

Johannes Angermuller, «Analyser le discours politique en Allemagne (1980-2010) », Mots. Les

langages du politique [En ligne], 94 | 2010, mis en ligne le 06 novembre 2012, consulté le 28 avril 2022 URL : http://journals.openedition.org/mots/19884 ; DOI : https://doi.org/10.4000/mots. 19884 


\section{Analyser le discours politique en Allemagne (1980-2010)}

\section{Les années soixante-dix, une période mouvementée}

Les années soixante-dix en Allemagne sont une période pleine de débats, de conflits et de contestation. C'est à la lumière de l'héritage des nazis, des divisions politiques et idéologiques de la Guerre froide, des nouveaux mouvements sociaux (féminisme, écologie, pacifisme...) que l'intérêt s'éveille pour l'analyse du discours politique, à l'Ouest (cf. la pragmatique du langage politique de Diekmann, 1969) pas moins qu'à l'Est (voir le modèle cybernétique de la propagande de Klaus, 1971).

En mettant l'accent sur les questions normatives et éthiques, l'École de Francfort est sans doute le phénomène intellectuel qui marque le plus l'intérêt naissant pour le discours politique. Pendant les années soixante-dix, ses théoriciens commencent leur «tournant linguistique » qui les mène des réflexions sur la langue allemande compromise par Auschwitz (Adorno) à la question d'une éthique discursive basée sur les lois universelles de la communication (Habermas, 1987). Ainsi, pour Habermas, le langage est moins un instrument «idéologique » cachant un rapport de forces sociales donné que la condition préalable pour entamer un procès de délibération qui permet de reconsidérer les pouvoirs et traditions en place. C'est ainsi qu'après les interrogations marxistes, Habermas prône une démarche plus consensualiste tout en privilégiant l'échange du meilleur argument dans l'espace public et le libre débat entre les citoyens.

\section{L’analyse du discours politique depuis les années quatre-vingt. Les évolutions en linguistique}

Si, au début des années quatre-vingt, avec l'arrivée au pouvoir de Helmut Kohl, les ambitions de la contestation politique se calment, le nouveau champ d'analyse du discours politique est consolidé. Venant pour la plupart des sciences du langage, quelques-uns des premiers spécialistes de l'analyse du 
discours politique semblent encore prolonger le programme marxiste d'une "critique de l'idéologie», notamment Siegfried Jäger avec son Institut de la recherche en sciences du langage et sciences sociales à Duisburg (DISS). Pionniers de «l'analyse du discours critique» (KDA) en Allemagne, Jäger et son équipe ont publié de nombreuses études sur le racisme et l'antisémitisme dans le discours médiatique. Alors que Jäger met sur son drapeau plutôt Foucault que Marx, Adorno ou Habermas, son objectif est de révéler les structures d'exclusion et de discrimination et ainsi d'intervenir dans le débat politique (Jäger, 2007). Avec son insistance sur la dimension du pouvoir, le DISS a suscité non seulement des critiques des linguistes plus disciplinaires, mais aussi de quelques acteurs du discours médiatique étonnés de se voir attribuer l'étiquette d'antisémite. Si Jürgen Link, son ami de longue date, partage cet accent sur le pouvoir, Link s'intéresse d'avantage à la symbolisation de l'ordre social. Avec son concept de «normalisme» et de «symbole collectif» (1982), Link vise à rendre compte des représentations symboliques de l'ordre social, par exemple d'une société avec un centre légitime et dominant («le peuple honnête ») et une périphérie plus ou moins «problématique» («les immigrés dangereux»). Tout comme Jäger, Link rejette la perspective micro qui ignorerait les inégalités. Il considère le discours comme un «interdiscours » (le discours politique dans son intégralité) organisé à la fois de façon verticale (entre discours dominants et dominés) et de façon horizontale (entre discours différenciés ou «spéciaux»).

Néanmoins, Jäger et Link ne sont pas les seuls à promouvoir l'analyse du discours. Si le DISS a jeté les bases de l'analyse du discours critique en Allemagne, la problématique du discours a également été découverte par des linguistes plus descriptifs. C'est dans le sillage de l'histoire de la langue que Dietrich Busse esquisse la théorie d'une sémantique historique (Busse, 1987) basée sur une pragmatique de l'agir communicationnel et inspirée par l'histoire conceptuelle. En s'appuyant sur Michel Foucault, il rédigera avec Wolfgang Teubert le programme pour une analyse du discours (Busse, Teubert, 1994) qui donnera suite à l'émergence de la linguistique discursive (Warnke éd., 2007). Alors que Busse examine la carrière historique de mots comme Nation («nation ») ou Gewalt («violence »), son collègue Martin Wengeler avance une approche argumentative du discours qui lui permet de révéler l'enchaînement des topoï dans un corpus portant sur l'immigration en Allemagne, de 1960 à 1985 (Wengeler, 2003). Étant donné l'accent mis sur le savoir, il est peu surprenant de voir un rapprochement grandissant avec la linguistique cognitive, notamment dans le travail d'Alexander Ziem sur les frames et les métaphores politiques comme celle de la sauterelle (Heuschrecke) pour les spéculateurs à la Bourse (2008). 


\section{Du macro au micro. Les tendances d'analyse du discours en sciences sociales}

Dans les sciences sociales, le discours désigne pendant longtemps un problème plutôt théorique dans la lignée d'Habermas. Cependant, autour de l'an 2000 , la réception de Foucault commence à mettre un terme à l'hégémonie habermasienne tout en créant un nouveau champ de recherche à la fois théorique et empirique. Habermas est critiqué par les intellectuels autour du "poststructuralisme », ces représentants de la French Theory qui reçoivent les théoriciens et intellectuels phares des années soixante-dix en France (Angermüller, 2007a). Les premiers à adopter cette nouvelle conception de discours (qu'on peut résumer par la formule discours = savoir + pouvoir) sont des féministes foucaldiennes et déconstructivistes qui, comme Judith Butler, rejettent toute notion d'une identité sexuelle basée sur une différence biologique donnée (Feministische Studien, 1993). Si Foucault était pendant longtemps campé aux marges de l'institution académique, il devient maintenant une référence incontournable qui permet à l'analyse du discours de se constituer comme un champ plus ou moins cohérent et intégré.

Ainsi, à la fin des années quatre-vingt-dix, l'analyse du discours fait-elle son entrée dans la sociologie, notamment dans la recherche qualitative qui était née pendant les années soixante-dix de la rencontre entre le pragmatisme américain (George Herbert Mead, Erving Goffman, Harold Garfinkel, etc.) et la tradition interprétative et herméneutique allemande (comme par exemple Max Weber et Alfred Schütz). Ainsi, Schwab-Trapp propose-t-il d'articuler le cadre d'interprétation de «l'herméneutique objective» - l'analyse séquentielle défendue par Ulrich Oevermann - avec un cadre foucaldien dans le but de rendre compte du discours d'après-guerre sur le nazisme (SchwabTrapp, 1996). De même, le groupe d'Augsbourg a rapproché la sociologie de la connaissance luckmannienne avec Foucault en créant «l'analyse du discours dans la lignée de la sociologie de la connaissance » (Wissenssoziologische Diskursanalyse, WDA) (Keller, 2001). Ainsi, dans une étude traitant le discours sur la «poubelle» en Allemagne et en France, Reiner Keller cite-il les outils interprétatifs de la recherche qualitative, notamment les « schèmes d'interprétation » (Deutungsmuster) afin de rendre compte du sens partagé de façon intersubjective dans une société (1998).

En même temps, d'autres courants se sont développés, moins tributaires de la tradition herméneutique et plus orientés vers le décentrement du sujet maître et autonome du sens (Angermüller, Bunzmann et Nonhoff, 2001). Ce sont les chercheurs inspirés par quelques théoriciens du (post)structuralisme tels que Michel Foucault, Jacques Derrida, Jacques Lacan, Judith Butler et Ernesto Laclau qui ont été canonisés depuis les années quatre-vingt-dix. Ce dernier est 
la référence pour le courant «analyse des hégémonies » défendu par le politologue Martin Nonhoff. Nonhoff analyse le discours sur l'économie sociale de marché - l'ordolibéralisme des premières décennies de la République fédérale - en traçant la logique laclausienne de la différence et de l'équivalence à travers de laquelle se constitue le débat public (Nonhoff, 2007). Cette perspective néo- ou poststructuraliste est également à la base de quelques études en géographie, la particularité de celles-ci étant de mobiliser les outils de la lexicométrie (Lexic03); voir notamment Georg Glasze, qui porte son regard sur la francophonie (Glasze, 2010), et Annika Mattissek qui étudie les représentations des villes dans la presse écrite (Mattissek, 2008). Cette dernière reprend également la problématique de la gouvernementalité, notion élaborée par Foucault dans ses cours au Collège de France entre 1976 et 1978 et reprise à la fin des années quatre-vingt-dix dans le cadre de la critique du néolibéralisme (Bröckling, Krasmann et Lemke, 2000). Autour de la gouvernementalité se forme ainsi un champ d'études ayant pour objet les manières d'exercer le pouvoir dans les sociétés libérales: de façon indirecte plutôt que de façon directe, par « la conduite des conduites» ou par «le gouvernement de soi-même» plutôt que par la commande et la discipline. C'est un pouvoir qui traverse les appareils institutionnels en déployant à la fois l'agir, le savoir et le parler (Angermüller, Van Dyk, 2010). En prolongeant cette perspective, Andrea Bührmann et Werner Schneider ouvrent l'analyse du discours à la problématique du dispositif et ses pratiques (Bührmann et Schneider, 2008).

Après les macro-approches de discours en sciences sociales, il faut mentionner les perspectives constructivistes plus «micro», plus proches des approches interactionnistes et conversationnelles. En guise d'exemple, je me permets de citer mes propres travaux sur le discours politique qui, grâce aux outils de la pragmatique énonciative, visent à dissocier les différentes voix et les différents niveaux énonciatifs du langage politique afin de révéler les divisions du sujet politique et ainsi l'hétérogénéité du sens (Angermüller, 2007b). Dans cette lignée, Jens Maeße, avec son étude sur le «processus de Bologne», montre la pluralité des voix plus ou moins anonymes qui animent ce discours technocratique sans sujet politique en traversant les différents niveaux et champs d'action politiques (Maeße, 2010). Si ces analyses considèrent le sens comme le produit instable de textes énoncés dans certains contextes, la rencontre avec l'ethnographie peut s'avérer particulièrement productive, comme en témoignent les travaux de Daniel Wrana et d'Antje Langer sur l'héritage nazi dans les institutions éducatives (Langer, Wrana 2009). Alors que ces approches insistent sur l'hétérogénéité du discours, sa fluidité et ses divisions, elles mettent en valeur l'activité des participants du discours. Le discours n'existe pas en dehors des usages qu'en font les individus dans des contextes spécifiques. Ce n'est pas une entité constituée, mais toujours à constituer par ceux qui se l'approprient. 


\section{L'analyse du discours politique en Allemagne aujourd'hui. Caractéristiques et perspectives}

Étant donné la panoplie de ces tendances, quelle est la spécificité, si l'on peut dire, de l'analyse du discours politique en Allemagne? En ce qui concerne les thèmes, on ne peut pas ne pas remarquer l'intérêt porté au nazisme, à l'environnement et au néolibéralisme, ce dernier étant devenu l'objet d'un nouveau champ d'études, celui de la gouvernementalité. En ce qui concerne les corpus, on préfère sans doute les corpus ouverts et hétérogènes, parfois codés par l'analyste à l'aide d'un logiciel d'analyse qualitative comme MaxQDA ou Altas.ti (mais voir le travail lexicométrique d'Annika Mattissek, Georg Glasze et Ronny Scholz). En ce qui concerne les méthodes, on remarque l'influence de la recherche qualitative qui, avec sa démarche interprétative, vise à rendre compte des pratiques et du sens vécu plutôt que de l'organisation formelle du matériel linguistique. En ce qui concerne les enjeux théoriques et épistémologiques, un problème privilégié est sans doute celui du savoir, d'un savoir passant du niveau national jusqu'au quotidien, des grandes collectivités jusqu'à l'individu.

Situé au carrefour de champs et traditions différents, le discours reste parfois une problématique floue, davantage un effet de mode que l'expression d'une réflexion approfondie. Souvent on voit une combinaison surprenante entre des traditions intellectuelles différentes : l'herméneutique et l'historiographie allemande, l'interactionnisme et les études culturelles américains, la critique structuraliste du sujet parlant telle qu'on la connaît en France, et tout ça avec Foucault comme figure surplombante. Ce dernier est devenu une référence pour une panoplie de tendances différentes : pour l'analyse du discours critique qui, à la différence de ses homologues autrichiennes et britanniques, se réclame de la critique foucaldienne du pouvoir (par exemple Jäger, Link); pour l'analyse du discours dans la lignée de la sociologie de la connaissance, qui se distingue avec Foucault des approches micro du discours (par exemple Keller), pour l'analyse du discours poststructuraliste qui cite Foucault comme épistémologue antihumaniste (par exemple Nonhoff, Mattissek, Glasze, Angermüller), pour l'analyse énonciative du discours qui s'appuie sur L'Archéologie du savoir afin de rendre compte de l'hétérogénéité du sens (par exemple Maeße, Mattissek, Angermüller).

Cette impureté, cette hybridité, ce mélange de traditions sont-ils à regretter? Dans la mesure où l'analyse du discours politique vise un objet complexe et hétérogène, ceci ne constitue pas forcément son point le plus faible. L'éternelle question n'est-elle pas plutôt : comment rendre compte d'un objet qui peut exister à l'écrit ainsi qu'à l'oral, d'un objet qui peut consister en des genres et des supports différents, de textes «matériels» et de pratiques sociales 
dotées de sens, d'un objet qui naît de l'association de textes et de contextes? C'est la rencontre de théories et méthodes différentes qui ouvre de nouvelles perspectives dans cette conjoncture que connaît l'analyse du discours politique en Allemagne actuellement ${ }^{1}$.

\section{Références}

ANGERMÜLLER J., 2007a, "Qu'est-ce que le “poststructuralisme français” ? À propos de la réception des tendances françaises de l'analyse du discours en Allemagne », Langage et société, nº 120, p. 17-34.

- 2007b, Nach dem Strukturalismus. Theoriediskurs und intellektuelles Feld in Frankreich, Bielefeld, Transcript.

Angermüller J., Bunzmann K., Nonhoff M. éd., 2001, Diskursanalyse : Theorien, Methoden, Anwendungen, Hambourg, Argument.

ANGERMÜLLER J., VAN DYK S. éd., 2010, Diskursanalyse meets Gouvernementalitätsforschung, Francfort-sur-le-Main, Campus.

Bröckling U., KRASMAN S., LEMKE T. éd., 2000, Gouvernementalität der Gegenwart, Francfort-sur-le-Main, Suhrkamp.

Bührmann A. D., Schneider W., 2008, Vom Diskurs zum Dispositiv. Eine Einführung in die Dispositivanalyse, Bielefeld, Transcript.

BUSSE D., 1987, Historische Semantik. Analyse eines Programms, Stuttgart, Klett-Cotta.

Busse D., TeUBeRT W., 1994, «Ist Diskurs ein sprachwissenschaftliches Objekt? Zur Methodenfrage der historischen Semantik», Begriffsgeschichte und Diskursgeschichte. Methodenfragen und Forschungsergebnisse der historischen Semantik, D. Busse, F. Hermanns, W. Teubert éd., Opladen, Westdeutscher Verlag, p. 10-28.

Diekmann W., 1969, Sprache in der Politik. Einführung in die Pragmatik und Semantik der politischen Sprache, Heidelberg, C. Winter.

Feministische Studien, 1993, n² 2 (11), Kritik der Kategorie « Geschlecht», numéro spécial. Glasze G., 2010, Politische Räume. Die diskursive Konstitution eines «geokulturellen Raums ». Die Frankophonie, Bielefeld, Transcript.

HABERMAS J., 1987, Théorie de l'agir communicationnel, Paris, Fayard.

JÄGER S., 2007 [1993], Kritische Diskursanalyse. Eine Einführung, Münster, Unrast.

KelLer R., 1998, Müll. Die gesellschaftliche Konstruktion des Wertvollen, Opladen, Westdeutscher Verlag.

- 2001, "Wissenssoziologische Diskursanalyse», Handbuch Sozialwissenschaftliche Diskursanalyse, vol.l, Theorien und Methoden, R. Keller, A. Hirseland, W. Schneider, W. Viehöver éd., Wiesbaden, VS Verlag für Sozialwissenschaften, p. 113-144.

KLAus G., 1971, Sprache der Politik, Berlin, Deutscher Verlag der Wissenschaften.

1. La plupart des chercheurs cités dans cette contribution participent activement au DiskursNetz. Pour en savoir plus sur l'analyse du discours en Allemagne, voir le site trilingue [http://www. analysedudiscours.net]. Site consulté le 19 juillet 2010. 
LANGER A., WranA D., 2009, «Diskursanalyse in der Erziehungswissenschaft. Forschungsgegenstände, Problemstellungen, Methoden », Handbuch Qualitative Forschungsmethoden in der Erziehungswissenschaft, B. Prengel, A. Friebertshäuser éd., Weinheim, Juventa.

LINK J., 1982, «Kollektivsymbole und Mediendiskurse», KultuRRevolution, nº 1, p. 6-21.

MAESSE J., 2010, Die vielen Stimmen des Bologna-Prozesses. Zur diskursiven Logik eines bildungspolitischen Programms, Bielefeld, Transcript.

MATtISSEK A., 2008, Die neoliberale Stadt. Diskursive Repräsentationen im Stadtmarketing deutscher Großstädte, Bielefeld, Transcript.

Nonhoff M., 2007, «L'analyse hégémonique : à propos du développement récent de l'analyse de discours politique dans les sciences politiques», Langage et société, $\mathrm{n}^{\circ} 120$, p. $77-90$.

SCHWAB-TRAPP M., 1996, Konflikt, Kultur und Interpretation. Eine Diskursanalyse des öffentlichen Umgangs mit dem Nationalsozialismus, Opladen, Westdeutscher Verlag.

WARNKE I. éd., 2007, Diskurslinguistiknach Foucault: Theorie und Gegenstände, Berlin, New York, de Gruyter.

Wengeler M., 2003, Topos und Diskurs. Begründung einer argumentationsanalytischen Methode und ihre Anwendung auf den Migrationsdiskurs (1960-1985), Tübingen, Niemeyer.

ZIEM A., 2008, Frames und sprachliches Wissen. Kognitive Aspekte der semantischen Kompetenz, Berlin, New York, de Gruyter. 